\title{
Effect of the Electron Lenses on the RHIC Proton Beam Closed Orbit
}

X. Gu, Y. Luo, A. Pikin, M. Okamura, W. Fischer, C. Montag, R. Gupta, J. Hock, A. Jain, D. Raparia

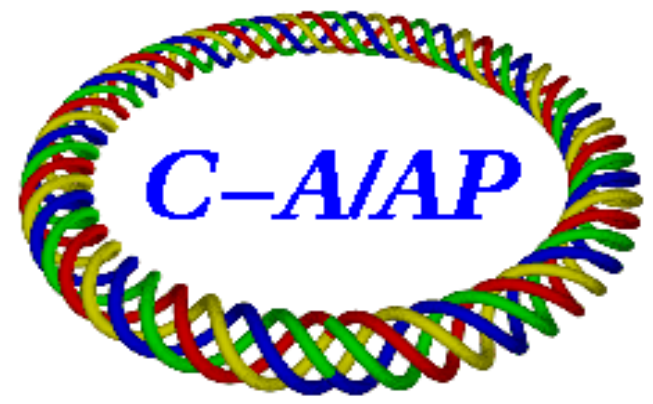

\section{Collider-Accelerator Department Brookhaven National Laboratory Upton, NY 11973}

Notice: This document has been authorized by employees of Brookhaven Science Associates, LLC under Contract No. DE-AC02-98CH10886 with the U.S. Department of Energy. The United States Government retains a non-exclusive, paid-up, irrevocable, world-wide license to publish or reproduce the published form of this document, or allow others to do so, for United States 


\title{
Effect of the Electron Lenses on the RHIC Proton Beam Closed Orbit
}

\author{
X. Gu, Y. Luo, A. Pikin, M. Okamura, W. Fischer, C. Montag, R. Gupta, J. Hock, A. Jain, D. \\ Raparia
}

\section{Brookhaven National Laboratory, Upton, NY 11973}

December 30, 2010

\begin{abstract}
We are designing two electron lenses (E-lens) to compensate for the large beam-beam tune spread from proton-proton interactions at IP6 and IP8 in the Relativistic Heavy lon Collider (RHIC). They will be installed at RHIC IR10. The transverse fields of the E-lenses' bending solenoids and the fringe field of the main solenoids will shift the proton beam. We calculate the transverse kicks that the proton beam receives in the electron lens via Opera. Then, after incorporating the simplified E-lens lattice in the RHIC lattice, we obtain the closed orbit effect with the Simtrack Code.
\end{abstract}

\section{Introduction}

The Relativistic Heavy Ion Collider (RHIC) at Brookhaven National Laboratory has operated for a decade. It has two rings in a horizontal plane with two head-on beam-beam interactions (IP6 and IP8) and four long-range beam-beam interactions.

The proton beam in the Blue ring circulates clockwise, while that in the Yellow ring circulates anticlockwise. In long-range beam-beam interactions the beam are separated vertically by $10 \mathrm{~mm}$. The current beam pipe at IP10 has a $65 \mathrm{~mm}$ radius.

Like in other colliders, the beam-beam interaction limits the luminosity of RHIC's polarized proton operation. To compensate for the large beam-beam tune spread due to head-on proton-proton interactions at IP6 and IP8 in RHIC, we are designing two E-lenses that we will install between the two DX dipoles at RHIC IR10. Here, we discuss the layout of these two E-lenses.

Furthermore, to clarify the effects of such electron lenses on RHIC's proton beam, we detail the layout of the RHIC lattice that includes the simplified E-lens lattice (Figure 1). Then, with this lattice in place, we evaluated, via the Simtrack code, the proton beam's closed orbit, its beta functions and its phase advance at IR10. 


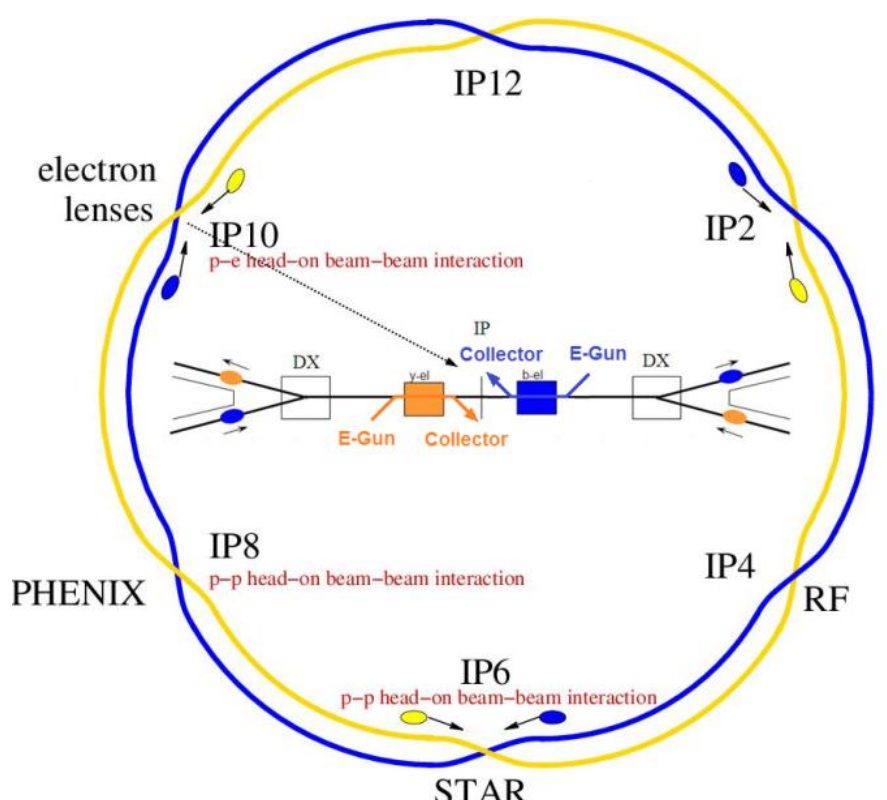

Figure 1: Layout of RHIC and of the E-lenses

\section{The Layout of the Two Electron Lenses}

Each of RHIC's E-lenses has one DC electron gun, one main superconducting magnet, one electron collector, and a beam transport system. This beam transport system has six solenoids, from the gun side to the collector side, viz., GS1, GS2, GSB, CSB, CS2, and CS1. To avoid affecting the DC electron guns with an unwanted electromagnetic field, we placed two DC electron guns away from the IP, while the two collectors are located near the IP (Figure 1).

Furthermore, to compensate for head-on beam-beam collision, the direction of the electron beam must be opposite that of the proton beam so that the electric and magnetic forces add. This means that when the E-lenses are operating, the ring's blue (yellow) proton beam must pass first through the yellow (blue) electron lenses. At this time, the blue (yellow) proton beam is being transported in the same direction as the yellow (blue) electron beam, but separated from it vertically by $10 \mathrm{~mm}$. Then, the blue (yellow) proton beam continues, progressing through the blue (yellow) electron lens, so that its transport direction is opposite that of the blue (yellow) electron beam.

Also, due to the $10 \mathrm{~mm}$ vertical separation between the two proton beams, the electron beams must be separated similarly. Two layouts of the E-lens can meet this requirement. First, we can set the vertical center of the two electron lenses at the same vertical position (i.e., the center of vacuum pipe, $Y=0$ ), and use steering magnets to move the electron beam either up by $5 \mathrm{~mm}$, or down by $5 \mathrm{~mm}$. We can achieve the same outcome mechanically, offsetting one of the electron lenses by $5 \mathrm{~mm}$ up from $\mathrm{Y}=0$, and the other $5 \mathrm{~mm}$ down. Then, the electron beam will interact head-on with the proton beam. The latter 
approach, the mechanical one, does not entail having magnets to move the electron beam $5 \mathrm{~mm}$ up and down, and so, it is easier to control.

Figure 2 shows the configuration of the magnetic structure of the two electron lenses with these constraints. The electron beam in one electron lens interacts head-on only with one proton beam.

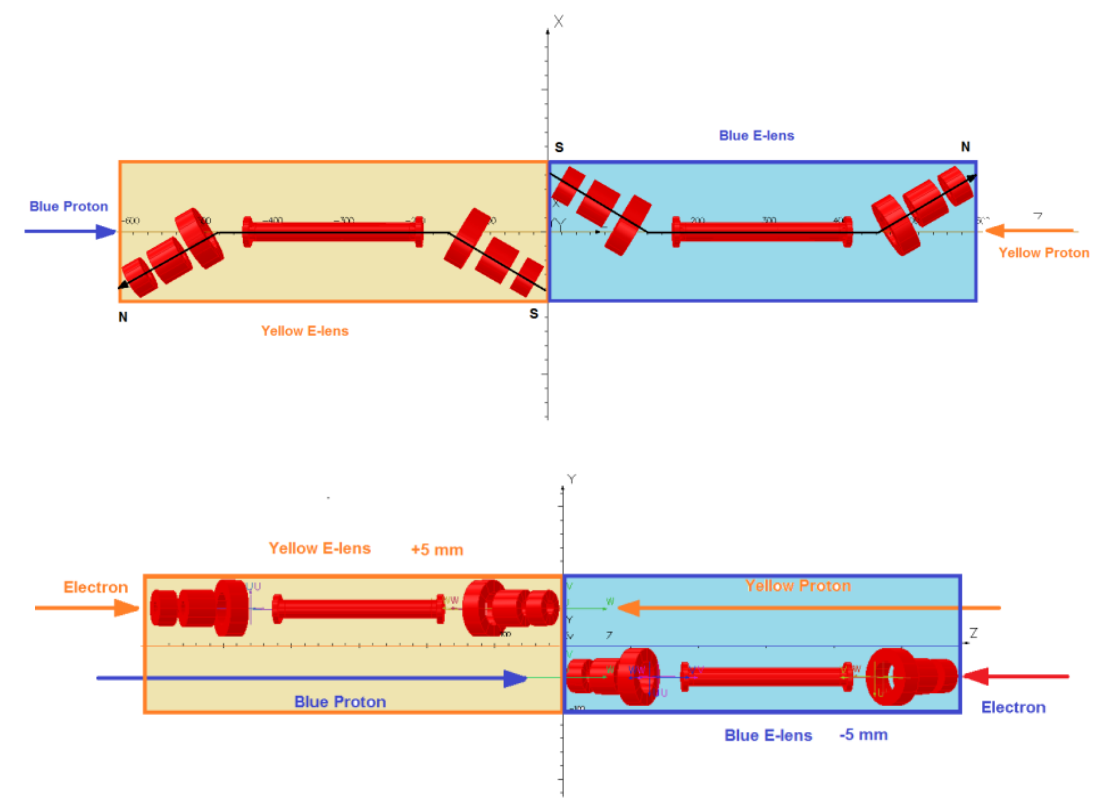

Figure 2: Layout of Two Electron Lenses. Top: top view, bottom: side view.

In addition to these constraints, the two lenses should have a different magnetic polarity (SN-NS or NS$\mathrm{SN}$ ) and therefore, locally compensate each other for both linear coupling and spin effects. Furthermore, the center line of the solenoids GSB_Y, CSB_Y, CSB_B, and GSB_B, located, respectively, at $-490 \mathrm{~cm},-145$ $\mathrm{cm}, 145 \mathrm{~cm}$, and $490 \mathrm{~cm}$ have a 30 degree angle between the directions of proton-beam transport. This configuration will induce high-order magnetic-field components when the proton beam passes through the two E-lenses; Figure 3 illustrates these components of two electron lenses along the center of the vacuum pipe.

The multipole magnetic field components in Fig. 3 generated by E-lens are analyzed and compared by Fourier fit method.

In cylindrical coordinates, we can express the radial and azimuthal components of magnetic field B in the form:

$$
\begin{aligned}
& \mathrm{B}_{\mathrm{r}}(\mathrm{r}, \theta)=\sum_{n=1}^{\infty}\left(b_{n} \sin (\mathrm{n} \theta)+a_{n} \cos (\mathrm{n} \theta)\right) \\
& \mathrm{B}_{\theta}(\mathrm{r}, \theta)=\sum_{n=1}^{\infty}\left(b_{n} \sin (\mathrm{n} \theta)-a_{n} \cos (\mathrm{n} \theta)\right)
\end{aligned}
$$

Where $b_{n}$ is the amplitudes of the $2 n$ pole normal term and $a_{n}$ is the amplitudes of $2 n$ pole skew term in the "European Convention". 
The multipole magnetic field, $B_{\theta}$ can be computed on a reference radius $R_{r e f}$ at different longitudinal positions and fitted as Fourier series. Then, according to formula (2), the coefficients of this Fourier series are the multipole magnetic field components. The reference radius $R_{r e f}=75 \mathrm{~mm}$.

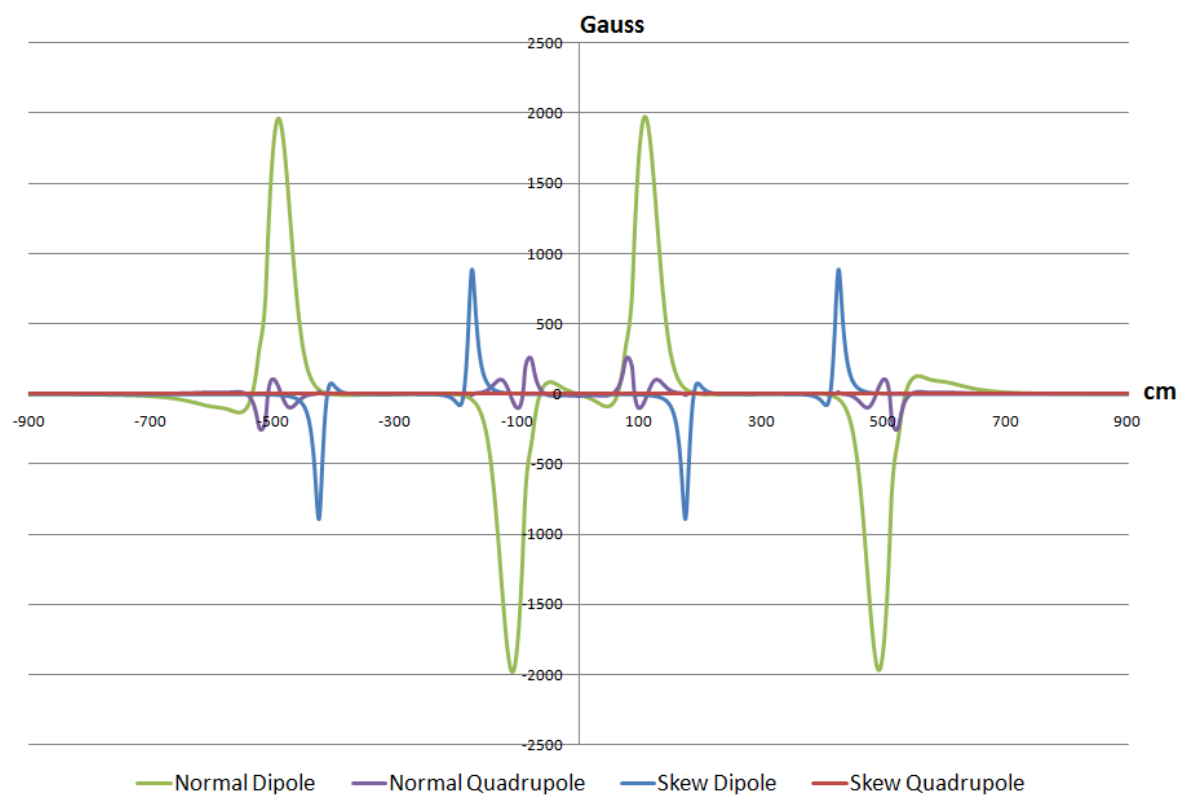

Figure 3: High-Order Magnetic Field Components.

\section{Single-pass Trajectories of the Proton Beam Tracked by Opera}

The dipole component field of the two electron lenses can deflect the trajectories of protons. To verify this effect and find a method to correct it, we tracked, by Opera, the centroid of the blue proton beam as it passed through these two electron lenses.

In our simulation, the blue proton beam starts from $-900 \mathrm{~cm}$ with eight different initial vertical angles; the horizontal angle is set to zero. The energy of the proton beam is $250 \mathrm{GeV}$, and the Lorentz factor is 266.

Figure 4 reveals that the blue proton beam has the same angle before and after the two electron lenses. However, within them, the blue proton beam is deflected. If its initial angle is about $100 \mu \mathrm{rad}$, the blue proton beams' trajectories within the two electron lenses can be set to be parallel to the Z-axis. 


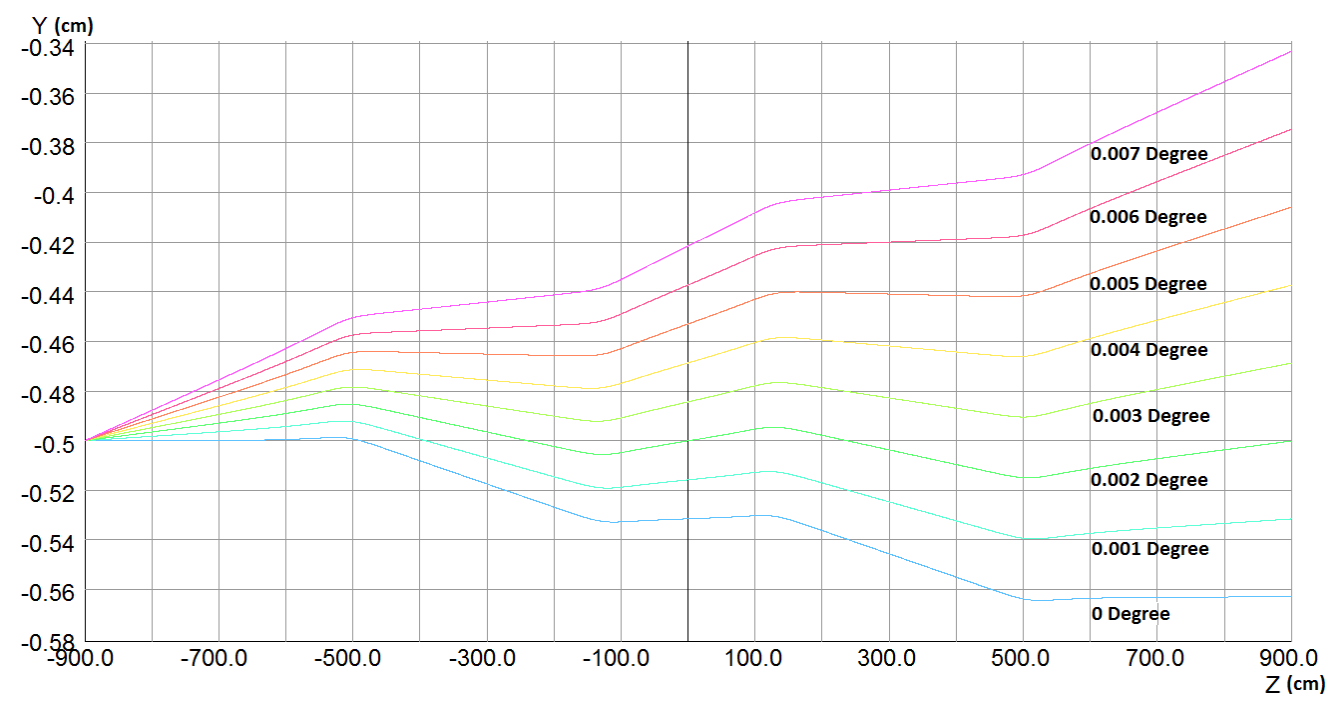

Figure 4: Proton Beam's Vertical Trajectories in Electron Lenses.

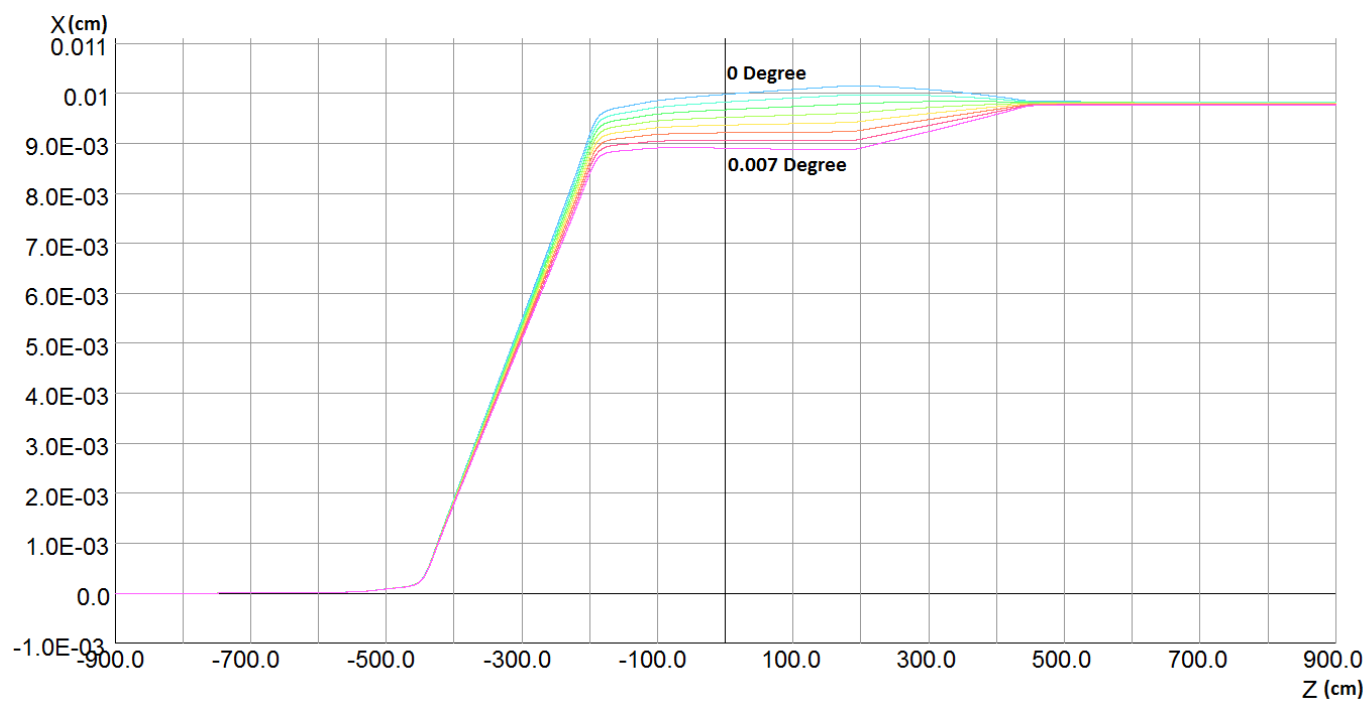

Figure 5: Proton Beam's Horizontal Trajectories in Electron Lenses.

Figure 5 plots the blue proton beam's horizontal trajectories. After passing through the two electron lenses, it exhibits a shift of about $0.01 \mathrm{~cm}$. Because the blue proton beam passes first through the yellow $5 \mathrm{~mm}$ E-lens, it is deflected by the fringe field of the yellow E-lens by about $0.009 \mathrm{~cm}$; this value is much greater than the change in the beam's position caused by the fringe field of the blue E-lens, which is less than $0.001 \mathrm{~cm}$.

\section{Simplification of the Electron Lenses' Lattice}

To include the E-lenses' elements lattice in the RHIC lattice, these elements, such as the transverse field of GSB and the fringe field of the main solenoid, must be simplified and replaced by an element that can be used in some tracking codes, such as Simtrack. 
In this paper, for simplification, we consider only the dipole components of the E-lenses' lattice, neglecting all higher order components.

Figure 6 and Figure 7 respectively, plot the transverse field of the GSB and the fringe field of main solenoid. In Figure 6 the horizontal field $B x$ was plotted for the line $(0,0, Z) \mathrm{cm}$ and $(1,1, Z) \mathrm{cm}$ (the red line); $Z$ is from $-700 \mathrm{~cm}$ to $-400 \mathrm{~cm}$. The two plots show only a slight difference. It is also confirmed that the vertical distribution of the $\mathrm{Bx}$ field does not change. Therefore, we assumed that $\mathrm{Bx}$ is constant in the XY plane.

In Figure 7 the vertical field By was plotted for the line $(0,-0.4, \mathrm{Z}) \mathrm{cm}$ (the red line) and $(0,-0.6, \mathrm{Z}) \mathrm{cm} ; \mathrm{Z}$ is from $-735 \mathrm{~cm}$ to $-435 \mathrm{~cm}$. When the elements of the electron lenses are simplified, By is also assumed to be uniform in the $\mathrm{XY}$ plane; even there is about $20 \%$ difference between $\mathrm{Bx}$ and $\mathrm{By}$ at the peak point of the field.

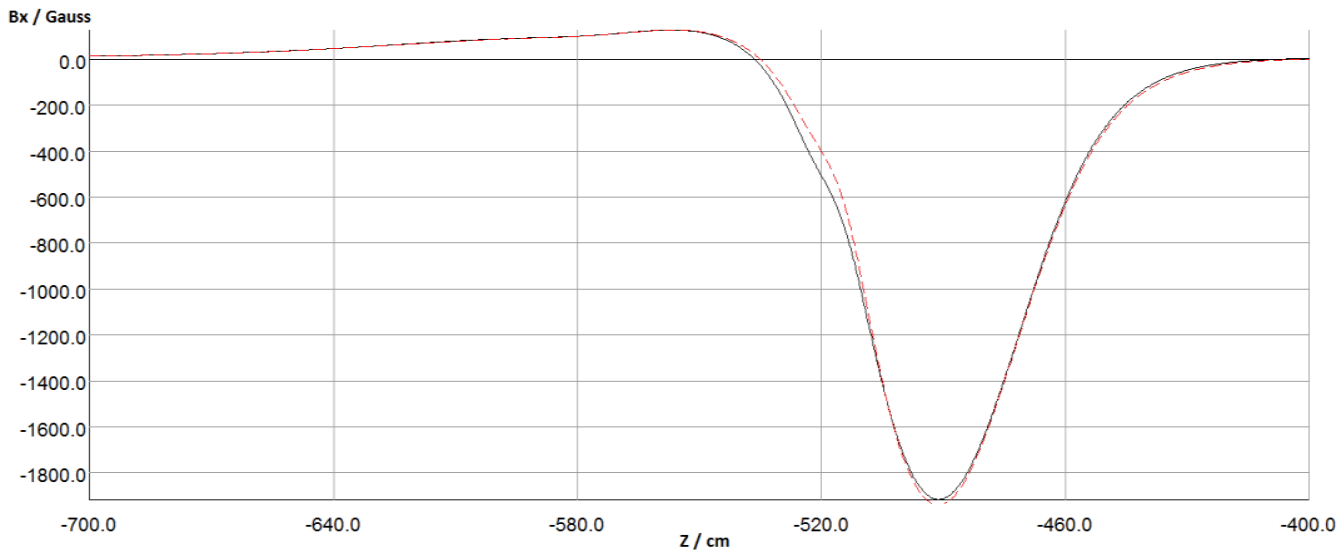

Figure 6: Bx field.

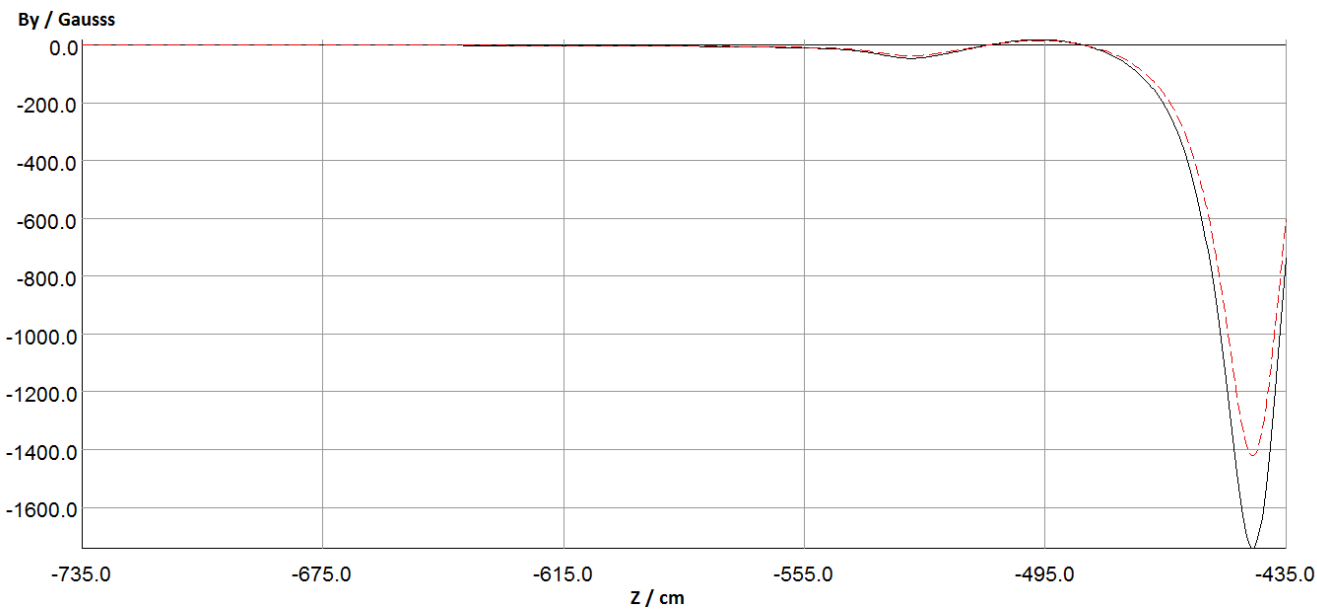

Figure 7: By Field

Since the fields $\mathrm{Bx}$ and $\mathrm{By}$ are assumed to be uniform fields in transverse plane, we also assumed that they are generated by many $X$ dipoles and $Y$ dipoles along the $Z$ axis. In the Simtrack code, we can 
represent the $X$ and $Y$ dipoles at the same longitudinal position by a KICKER element, which has a vertical and a horizontal kicker force.

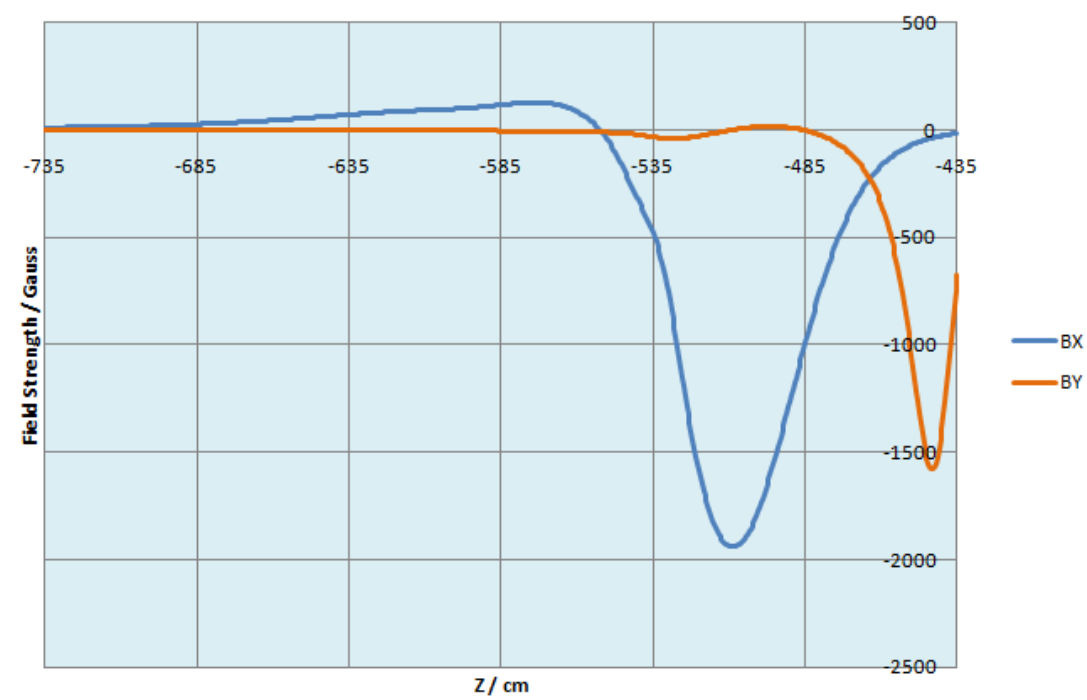

Figure 8: The Magnetic Field from $-735 \mathrm{~cm}$ to $-435 \mathrm{~cm}$.

Figure 8, Figure 9 and Figure 10, respectively, are the magnetic fields between $(-735,-435) \mathrm{cm},(-200$, 200) $\mathrm{cm}$, and $(435,735) \mathrm{cm}$ along $(0,-0.5, \mathrm{Z}) \mathrm{cm}$. In the Simtrack code, these magnetic fields are represented, separately, by 30-, 40-, and 30-KICKERs; each KICKER is $10 \mathrm{~cm}$ long.

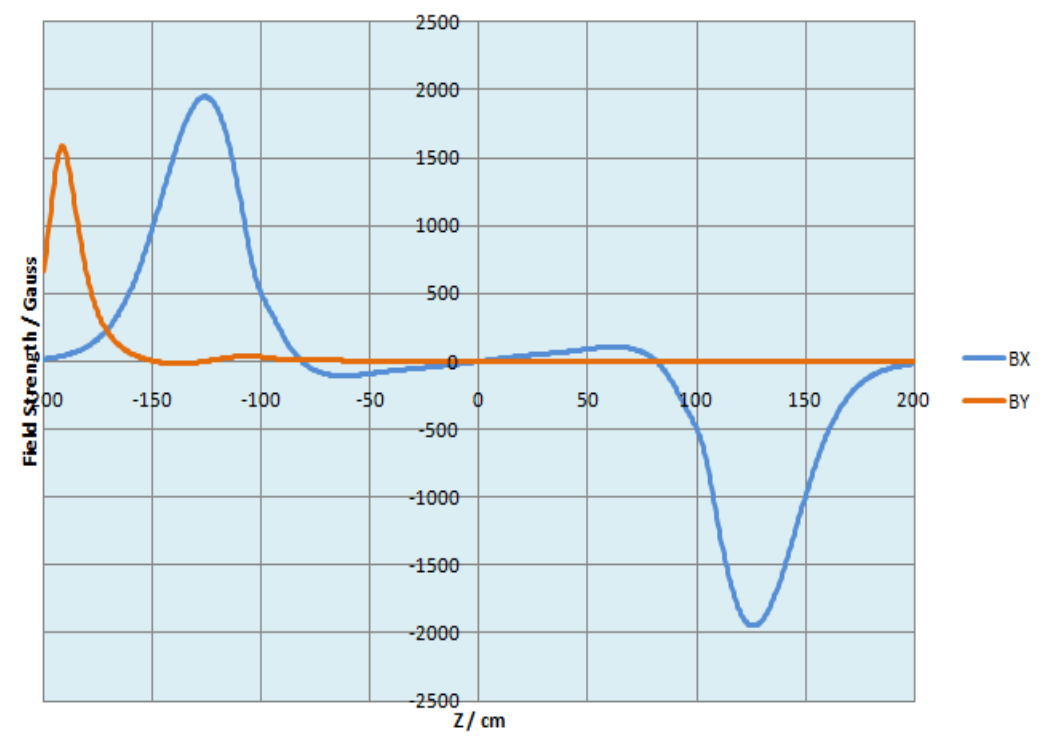

Figure 9: The Magnetic Field from $-200 \mathrm{~cm}$ to $+200 \mathrm{~cm}$ 


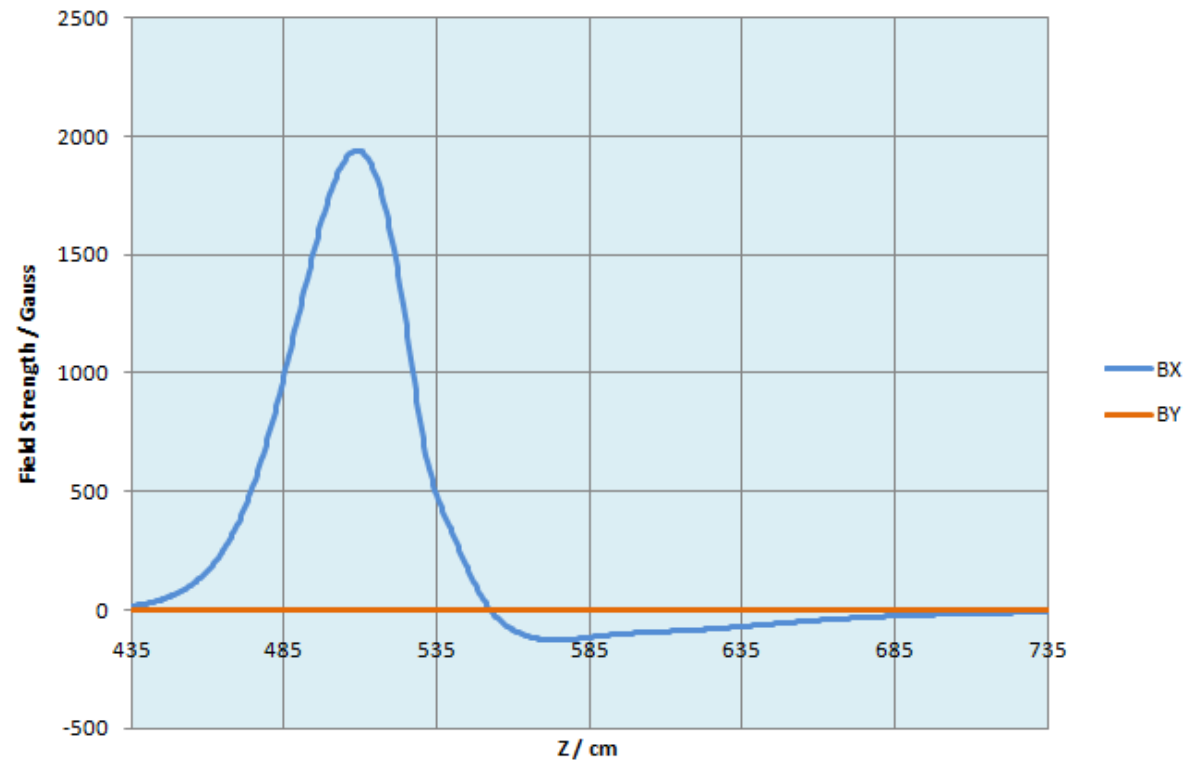

Figure 10: The Magnetic Field from $435 \mathrm{~cm}$ to $735 \mathrm{~cm}$.

Fig 11 is the layout of the electron lenses' lattice, which is about $7.412230 \mathrm{~m}$ and $7.412232 \mathrm{~m}$ long for left side of IP10 and right side of IP10. It replaces the element from AP10P4G to AP10P21G around IP 10. Because there is a vertical field, By, inside the main solenoid (due to a $5 \mathrm{~mm}$ offset), the length of this solenoid is reduced to $2.35 \mathrm{~m}$, but the integral values of $B z$ and $L$ remain constant; the 2.35 long solenoid has the same ks as the 2.5 m solenoids. Appendix A gives more details.

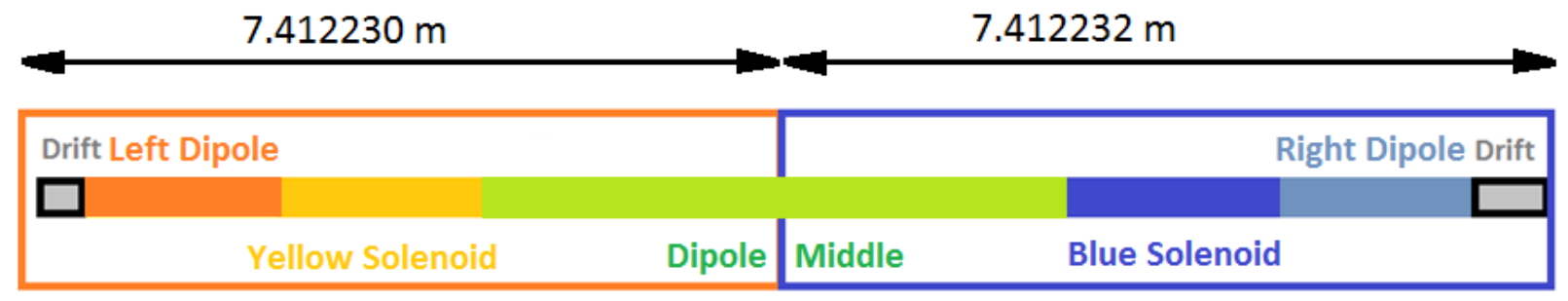

IP10

Figure 11: The layout of the electron lens lattice.

\section{Closed Orbit Calculation with Simtrack Code}

With the Simtrack code, we determined the entire ring's closed orbit including the RHIC lattice that encompasses the electron lenses' lattice: the results are shown in Figure 12 and Figure 13.

For tracking the 2011 Blue $250 \mathrm{GeV}$ polarized proton lattice was used. Its tune was set to $(28.67,29.675)$, and its chromaticity was set to $(1.0,1.0)$ without head-on beam-beam collisions at IP 6 and IP8. Because of this, the initial angle of the proton beam is set before it goes into the E-lens lattice, this is one reason that the proton beams have different shift between Opera and Simtrack code tracking. 
From Figure 12, the vertical proton beam's closed orbit changes $1.3 \mathrm{~mm}$ in $(-735,735) \mathrm{cm}$. IP 10 , with its zero coordinate, is the center of the E-lens. For the horizontal plane, the closed orbit changes $0.18 \mathrm{~mm}$. The green lines in Figure 12 and Figure 13 are the closed orbits without the E-lenses' lattice, which have zero transverse offset.
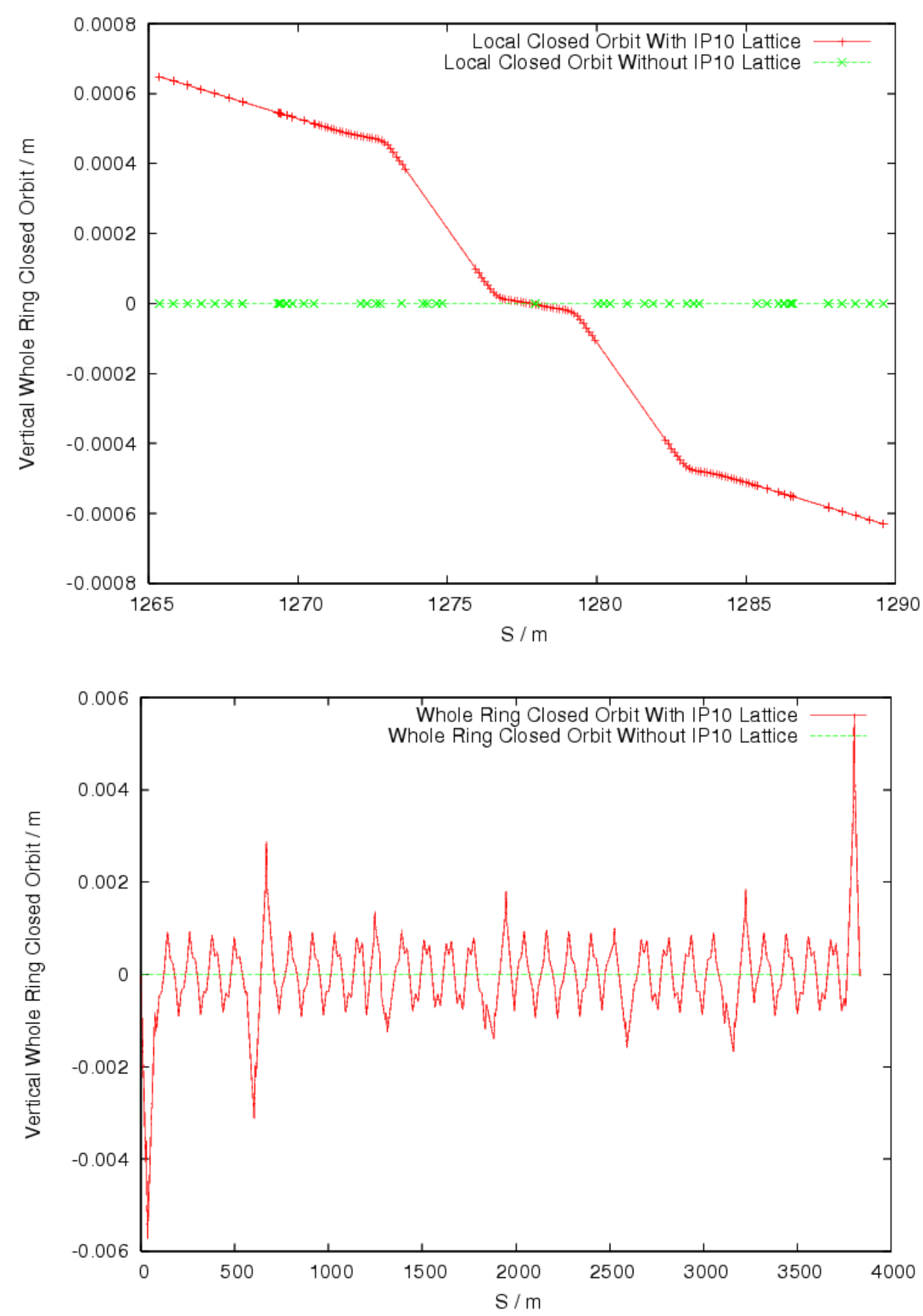

Figure 12: The Vertical Closed Orbit with (Red) and without (Green) the E-lens Lattice. 

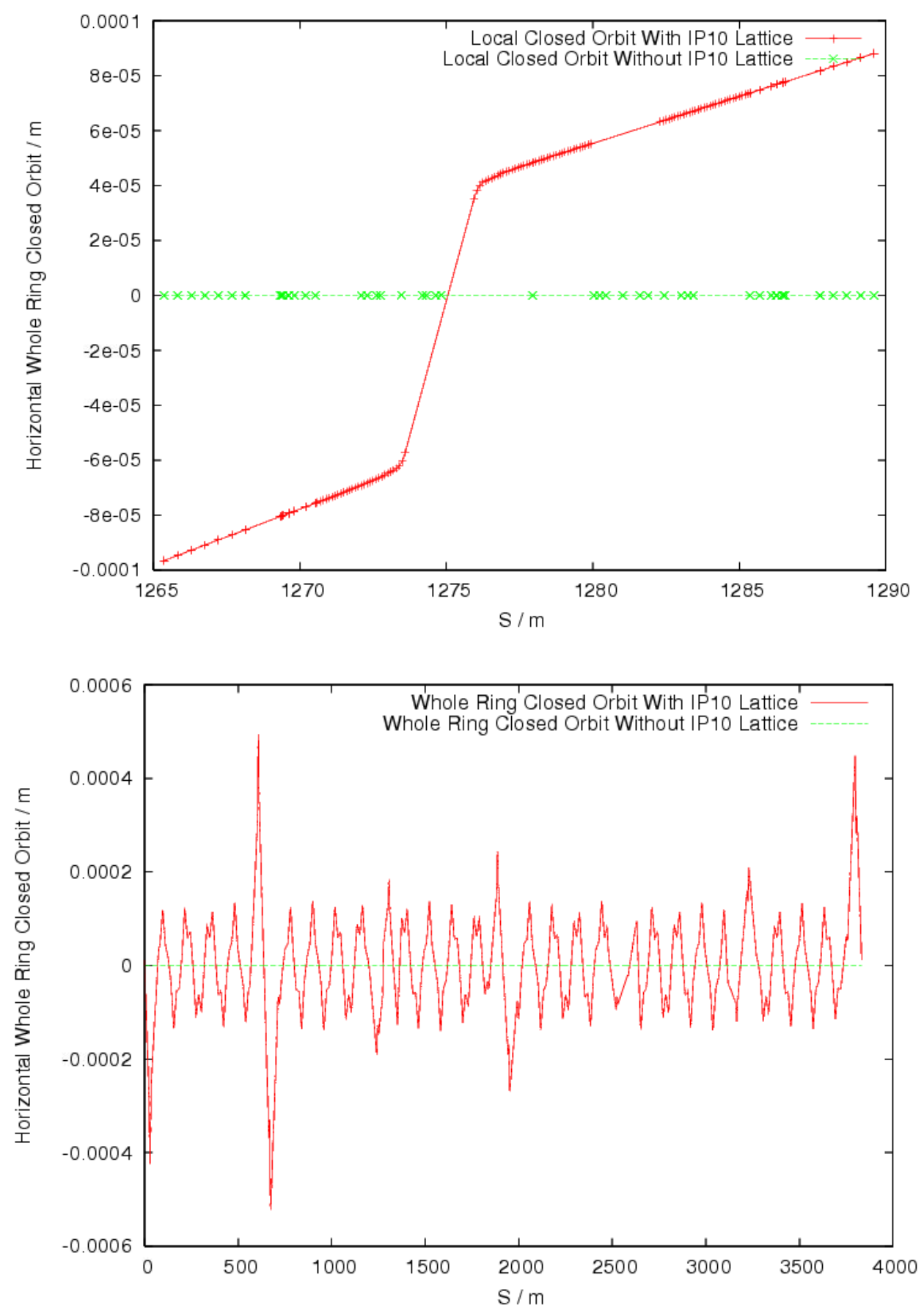

Figure 13: The Horizontal Closed Orbit with (Red) and without (Green) the E-lens Lattice.

Figure 14 and Figure 15 plot, respectively, the horizontal- and vertical-beta function with and without the E-lens lattice. They show no big differences because there is no large quadrupole error introduced. 


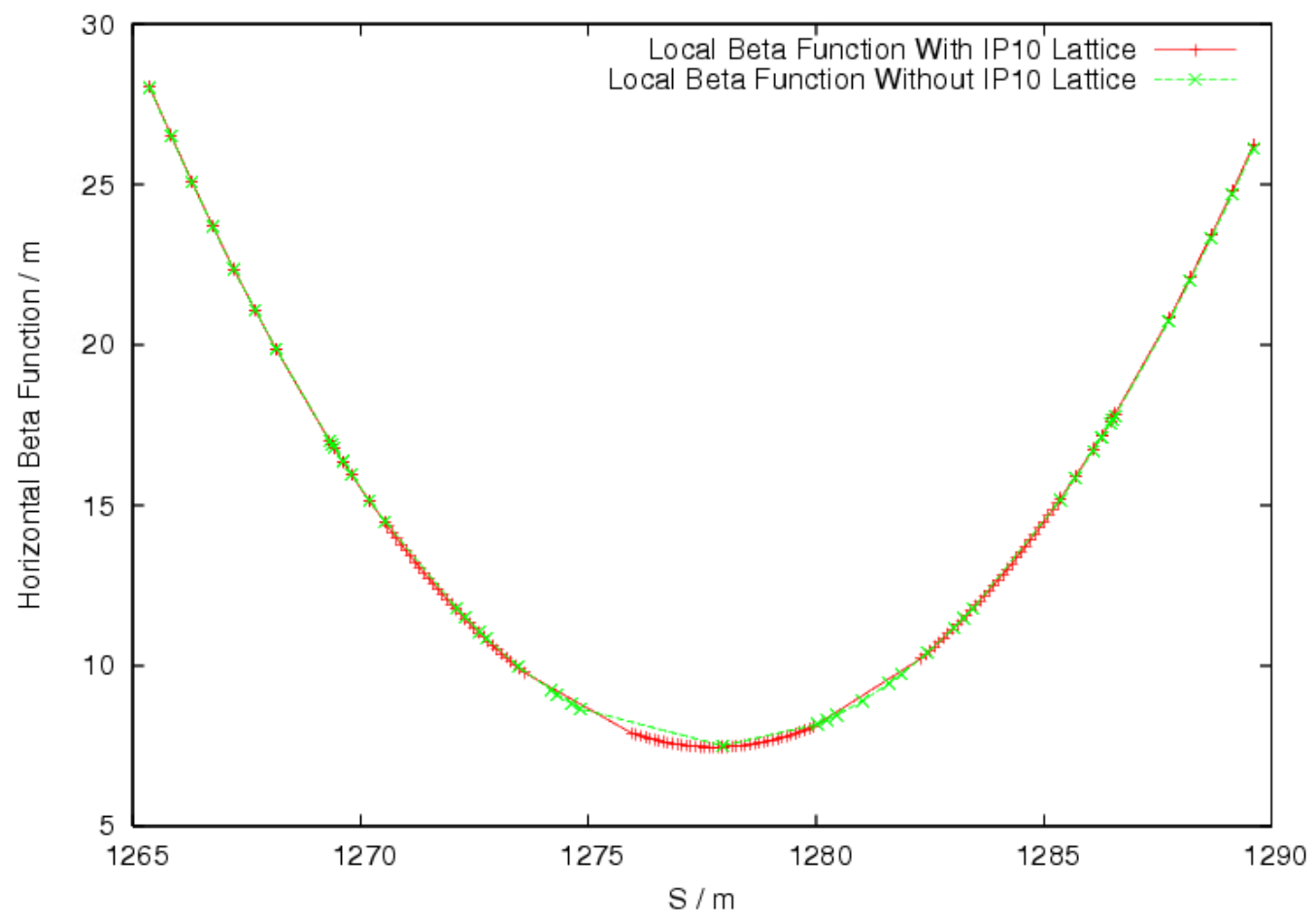

Figure 14: The Horizontal Beta Function with (Red) and without (Green) the E-lenses Lattice.

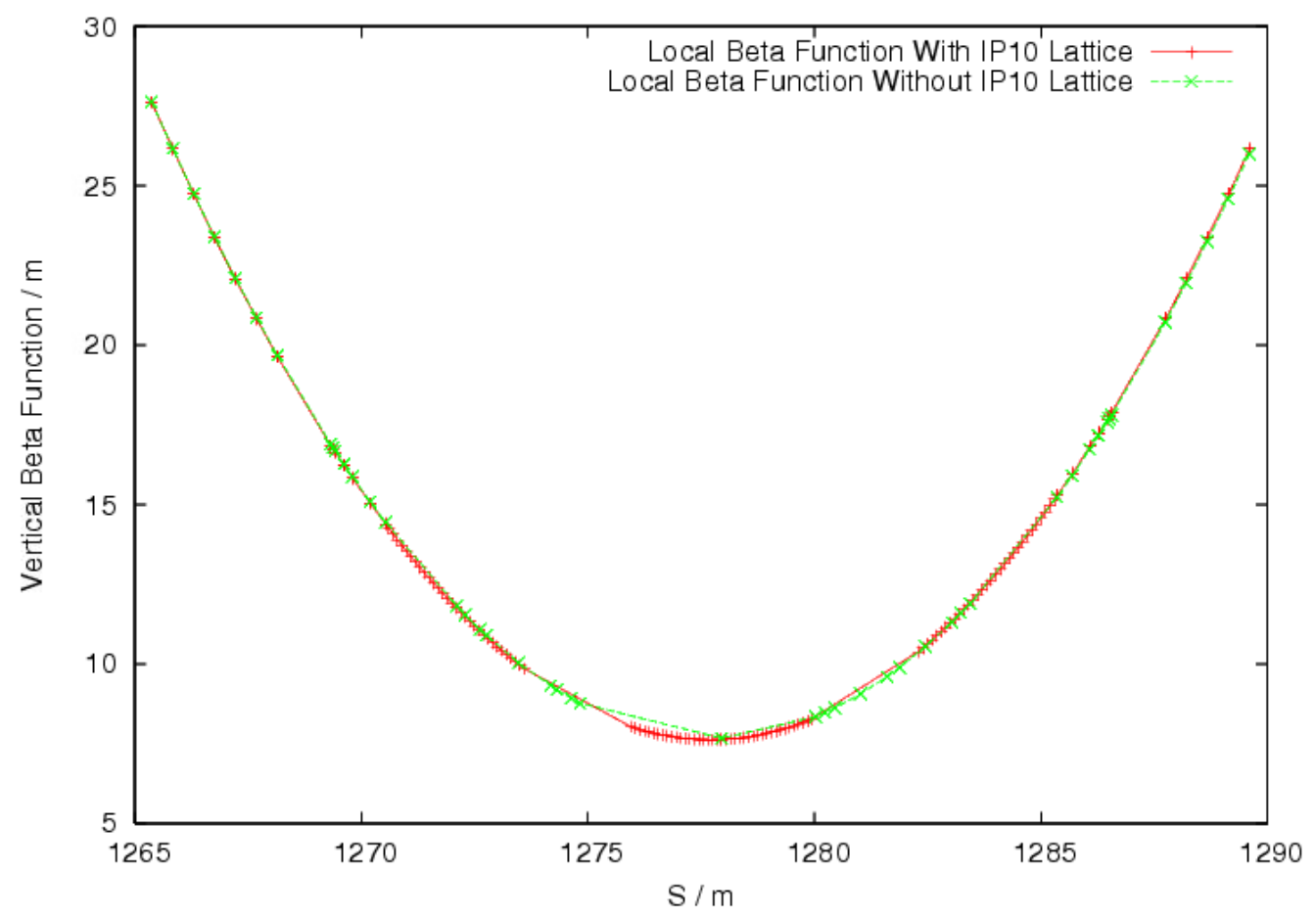

Figure 15: The Vertical Beta Function with (Red) and without (Green) the E-lenses Lattice.

Figure 16 illustrates the phase advance of the elements around IP10 with and without the E-lens for the vertical- and horizontal-plane. 


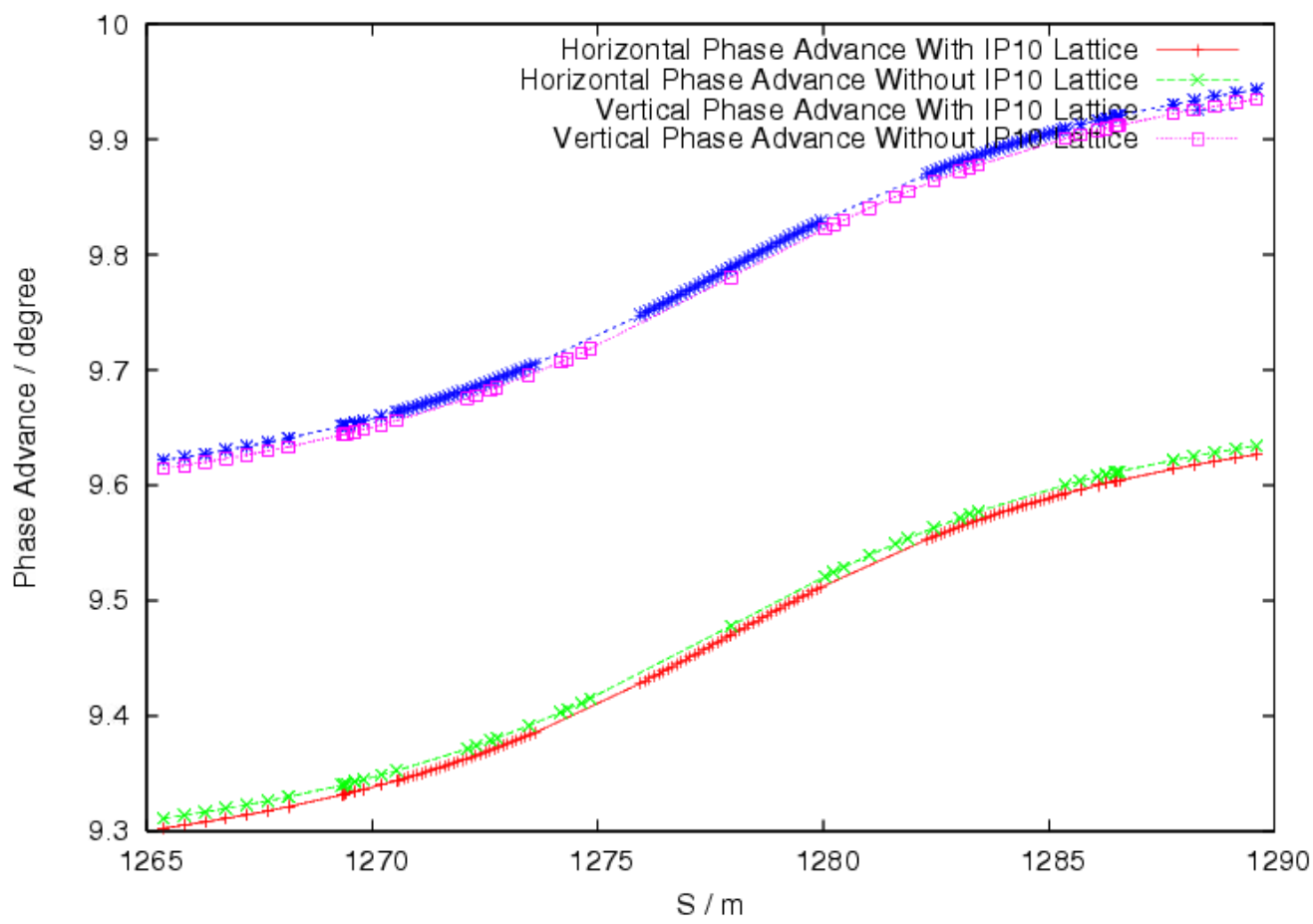

Figure 16: The Phase Advance with (Red and Blue) and without (Green and Magenta) the E-lens Lattice.

Table 1 lists the parameters for the proton beam with and without Elens lattice. The horizontal tune changed 0.0001 and vertical tune changed 0.0003 . Other parameters also have no big difference with and without Elens lattice.

Table 1 Proton Beam Parameters With and Without Elens.

\begin{tabular}{|c|c|c|c|c|}
\hline \hline \multirow{2}{*}{ Parameters } & \multicolumn{2}{|c|}{ Without Elens Lattice } & \multicolumn{2}{c|}{ With Elens Lattice } \\
\cline { 2 - 5 } & $\mathrm{X}$ & $\mathrm{Y}$ & $\mathrm{X}$ & $\mathrm{Y}$ \\
\hline Tune & 28.6949 & 29.6849 & 28.6950 & 29.6846 \\
\hline Chrom1 & 0.882692 & 0.91706 & 0.811907 & 0.905909 \\
\hline Chrom2 & 1867.42 & 1977.68 & 1871.67 & 1962.08 \\
\hline Alpha\# & 0.714805 & 0.708052 & 0.726546 & 0.71088 \\
\hline Beta\# & -0.144506 & -0.0409973 & -0.191133 & 0.00453062 \\
\hline
\end{tabular}

\# that is the twiss parameters at the starting point.

\section{Calculation of the E-lens Transport Matrix by Opera}

A third method of tracking the proton beam with the E-lenses is by the transport matrix, for example, from $-9 \mathrm{~m}$ to $9 \mathrm{~m}$ around IP10. We also can calculate the transport matrix from $-9 \mathrm{~m}$ to the inner side of main solenoids, such as $-4,-2,2$, and $4 \mathrm{~m}$. Knowing the initial proton beam parameters, we then can determine the beam's parameters inside the superconducting solenoid from these transport matrixes when the E-lens is operating. 
To assess these transport matrixes, we used the response matrix calculating method. One initial parameter is changed, for example, the $d x$ in the Table 2, and then Opera calculates the output value $d X$, $d X^{\prime}, d Y$, and $d Y^{\prime}$ as the function of $d x$. If these values display a linear function, we can fit them, as in the first column of Figure 17 (Appendix B). Then, we can obtain their coefficients from the fitting, as in the first column of Table.

Table 2: How to Get the Matrix Elements.

\begin{tabular}{|c|c|c|}
\hline Variable & Input & Output \\
\hline \multirow{4}{*}{$d x$} & $\mathrm{dx}$ & $d X / d x$ \\
\hline & 0 & $d X^{\prime} / d x$ \\
\hline & -0.005 & $d Y / d x$ \\
\hline & 0 & $d Y^{\prime} / d x$ \\
\hline \multirow{4}{*}{$d x^{\prime}$} & 0 & $d X / d x^{\prime}$ \\
\hline & $d x^{\prime}$ & $d X^{\prime} / d x^{\prime}$ \\
\hline & -0.005 & $d Y / d x^{\prime}$ \\
\hline & 0 & $d Y^{\prime} / d x^{\prime}$ \\
\hline \multirow{4}{*}{ dy } & 0 & $d X / d y$ \\
\hline & 0 & $\mathrm{dX}^{\prime} / \mathrm{dy}$ \\
\hline & dy-0.005 & $d Y / d y$ \\
\hline & 0 & $d Y^{\prime} / d y$ \\
\hline \multirow{4}{*}{$d y^{\prime}$} & 0 & $\mathrm{dX} / \mathrm{dy}$ \\
\hline & 0 & $\mathrm{dX}^{\prime} / \mathrm{dy} \mathrm{y}^{\prime}$ \\
\hline & -0.005 & $d Y / d y^{\prime}$ \\
\hline & $d y^{\prime}$ & $d Y^{\prime} / d y^{\prime}$ \\
\hline
\end{tabular}

Thereafter, we repeated the calculation for $d x^{\prime}, d y$, and $d y^{\prime}$ in Table 1 to get the other three columns in the Table 3. In this manner, we have all the transport matrix elements from -9 to $Z$ ( $Z$ may be inside or outside the E-lens).

Table3: The Matrix Elements.

\begin{tabular}{llll}
\hline \hline$d X / d x$ & $d X / d x^{\prime}$ & $d X / d y$ & $d X / d y^{\prime}$ \\
$d X^{\prime} / d x$ & $d X^{\prime} / d x^{\prime}$ & $d X^{\prime} / d y$ & $d X^{\prime} / d y^{\prime}$ \\
$d Y^{\prime} / d x$ & $d Y^{\prime} / d x^{\prime}$ & $d Y^{\prime} / d y$ & $d Y^{\prime} / d y^{\prime}$ \\
$d Y^{\prime} / d x$ & $d Y^{\prime} / d x^{\prime}$ & $d Y^{\prime} / d y$ & $d Y^{\prime} / d y^{\prime}$ \\
\hline \hline
\end{tabular}

For the transport matrix from $-9 \mathrm{~m}$ to $9 \mathrm{~m}$, the transport matrix is

$\begin{array}{rrrr}0.9994 & 17.99 & 1.00 \mathrm{E}-05 & 0.0044 \\ -7.00 \mathrm{E}-05 & 0.9993 & 1.00 \mathrm{E}-06 & -1.00 \mathrm{E}-05\end{array}$




$\begin{array}{rrrr}-8.00 \mathrm{E}-06 & -0.0044 & 0.9994 & -17.991 \\ -7.00 \mathrm{E}-07 & -5.00 \mathrm{E}-06 & -6.00 \mathrm{E}-05 & -1.00 \mathrm{E}+00\end{array}$

And the constant matrix is

0.000100

$-0.005629$

0

When we use these transport matrixes, the output parameters ([1*4] array) are equal to the transport matrix $([4 * 4]$ array) times the input parameters ([1*4] array) plus a constant matrix ([1*4] array).

\section{Discussion}

In this paper, we discuss our design of the vertical- and horizontal-layout of two RHIC electron lenses. Firstly, we simulated, with Opera, the single pass trajectories of the proton beam in these two electron lenses. With about a 100 - $\mu$ rad initial vertical angle, the proton beam becomes parallel to the Z-axis that is, the axis of the electron beam.

An E-lens lattice is created without considering the higher order magnetic field, and misalignments of its elements. Further, we tracked by the Simtrack code, the entire ring's beam parameters with the 2011 blue $250-\mathrm{GeV}$ polarized proton beam's lattice that includes the E-lens lattice.

Finally, we introduced the transport matrix method as the third method for tracking the proton beam.

These trackings and simulations give us a greater understanding about the proton beam's behavior inside the E-lens, and also afford clues on the alignment between the proton beam and the electron beam when we apply head-on beam-beam compensation. 


\section{Appendix A}

\begin{tabular}{|c|c|c|c|c|c|c|c|}
\hline \multicolumn{4}{|c|}{ IP 10 Lattice without E-lens } & \multicolumn{4}{|c|}{ IP 10 Lattice with E-lens } \\
\hline Element & Location & Length & Type & Element & Location & Length & Type \\
\hline HDXPM09 & 1265.37 & 0.462527 & SBEND & HDXPM09 & 1265.37 & 0.462527 & SBEND \\
\hline HDXPM09M2 & 1265.37 & 0 & MULT & HDXPM09M2 & 1265.37 & 0 & MULT \\
\hline HDXPM09 & 1265.84 & 0.462527 & SBEND & HDXPM09 & 1265.84 & 0.462527 & SBEND \\
\hline HDXPM09M3 & 1265.84 & 0 & MULT & HDXPM09M3 & 1265.84 & 0 & MULT \\
\hline HDXPM09 & 1266.3 & 0.462527 & SBEND & HDXPM09 & 1266.3 & 0.462527 & SBEND \\
\hline HDXPM09M4 & 1266.3 & 0 & MULT & HDXPM09M4 & 1266.3 & 0 & MULT \\
\hline HDXPM09 & 1266.76 & 0.462527 & SBEND & HDXPM09 & 1266.76 & 0.462527 & SBEND \\
\hline HDXPM09M5 & 1266.76 & 0 & MULT & HDXPM09M5 & 1266.76 & 0 & MULT \\
\hline HDXPM09 & 1267.22 & 0.462527 & SBEND & HDXPM09 & 1267.22 & 0.462527 & SBEND \\
\hline HDXPM09M6 & 1267.22 & 0 & MULT & HDXPM09M6 & 1267.22 & 0 & MULT \\
\hline HDXPM09 & 1267.69 & 0.462527 & SBEND & HDXPM09 & 1267.69 & 0.462527 & SBEND \\
\hline HDXPM09M7 & 1267.69 & 0 & MULT & HDXPM09M7 & 1267.69 & 0 & MULT \\
\hline HDXPM09 & 1268.15 & 0.462527 & SBEND & HDXPM09 & 1268.15 & 0.462527 & SBEND \\
\hline HDXPM09M8 & 1268.15 & 0 & MULT & HDXPM09M8 & 1268.15 & 0 & MULT \\
\hline ELDXPM09 & 1268.15 & 0 & DRIFT & ELDXPM09 & 1268.15 & 0 & DRIFT \\
\hline ODXFLX & 1269.33 & 1.18563 & DRIFT & ODXFLX & 1269.33 & 1.18563 & DRIFT \\
\hline AP10P0G & 1269.33 & 0 & MARKER & AP10P0G & 1269.33 & 0 & MARKER \\
\hline OBSK9LG & 1269.38 & 0.048623 & DRIFT & OBSK9LG & 1269.38 & 0.048623 & DRIFT \\
\hline MVALVE & 1269.38 & 0 & MARKER & MVALVE & 1269.38 & 0 & MARKER \\
\hline OX9A0 & 1269.43 & 0.04875 & DRIFT & OX9A0 & 1269.43 & 0.04875 & DRIFT \\
\hline AP10P1G & 1269.43 & 0 & MARKER & AP10P1G & 1269.43 & 0 & MARKER \\
\hline OBXFLA & 1269.62 & 0.1905 & DRIFT & OBXFLA & 1269.62 & 0.1905 & DRIFT \\
\hline BPMH & 1269.62 & 0 & HBPM & $\mathrm{BPMH}$ & 1269.62 & 0 & HBPM \\
\hline CPLMON & 1269.62 & 0 & BPM & CPLMON & 1269.62 & 0 & BPM \\
\hline BPMV & 1269.62 & 0 & VBPM & BPMV & 1269.62 & 0 & VBPM \\
\hline OBXFLX & 1269.81 & 0.1905 & DRIFT & OBXFLX & 1269.81 & 0.1905 & DRIFT \\
\hline AP10P2G & 1269.81 & 0 & MARKER & AP10P2G & 1269.81 & 0 & MARKER \\
\hline OX9A1 & 1270.2 & 0.390525 & DRIFT & OX9A1 & 1270.2 & 0.390525 & DRIFT \\
\hline AP10P3G & 1270.2 & 0 & MARKER & AP10P3G & 1270.2 & 0 & MARKER \\
\hline OX9A2 & 1270.54 & 0.333248 & DRIFT & OX9A2 & 1270.54 & 0.333248 & DRIFT \\
\hline AP10P4G & 1270.54 & 0 & MARKER & AP10P4G & 1270.54 & 0 & MARKER \\
\hline OX9A3 & 1272.11 & 1.56947 & DRIFT & LDFT_Yellow & 1270.6 & 0.06223 & DRIFT \\
\hline AP10P5G & 1272.11 & 0 & MARKER & Left Dipole & 1270.7 & 0.1 & KICKER \\
\hline OX9A4 & 1272.3 & 0.19812 & DRIFT & Left Dipole & 1270.8 & 0.1 & KICKER \\
\hline AP10P6G & 1272.3 & 0 & MARKER & Left Dipole & 1270.9 & 0.1 & KICKER \\
\hline
\end{tabular}




\begin{tabular}{|c|c|c|c|c|c|c|c|}
\hline OX9A5 & 1272.62 & 0.3175 & DRIFT & Left Dipole & 1271 & 0.1 & KICKER \\
\hline AP10P7G & 1272.62 & 0 & MARKER & Left Dipole & 1271.1 & 0.1 & KICKER \\
\hline OX9A6 & 1272.76 & 0.141986 & DRIFT & Left Dipole & 1271.2 & 0.1 & KICKER \\
\hline AP10P8G & 1272.76 & 0 & MARKER & Left Dipole & 1271.3 & 0.1 & KICKER \\
\hline OTWHFL1 & 1273.47 & 0.707009 & DRIFT & Left Dipole & 1271.4 & 0.1 & KICKER \\
\hline TWH & 1273.47 & 0 & DRIFT & Left Dipole & 1271.5 & 0.1 & KICKER \\
\hline OTWHFL2 & 1274.18 & 0.707009 & DRIFT & Left Dipole & 1271.6 & 0.1 & KICKER \\
\hline AP10P9G & 1274.18 & 0 & MARKER & Left Dipole & 1271.7 & 0.1 & KICKER \\
\hline OX9A7 & 1274.32 & 0.141986 & DRIFT & Left Dipole & 1271.8 & 0.1 & KICKER \\
\hline AP10P10G & 1274.32 & 0 & MARKER & Left Dipole & 1271.9 & 0.1 & KICKER \\
\hline OX9A8 & 1274.64 & 0.3175 & DRIFT & Left Dipole & 1272 & 0.1 & KICKER \\
\hline AP10P11G & 1274.64 & 0 & MARKER & Left Dipole & 1272.1 & 0.1 & KICKER \\
\hline OX9A9 & 1274.83 & 0.19812 & DRIFT & Left Dipole & 1272.2 & 0.1 & KICKER \\
\hline AP10P12G & 1274.83 & 0 & MARKER & Left Dipole & 1272.3 & 0.1 & KICKER \\
\hline OX9A10 & 1277.95 & 3.11353 & DRIFT & Left Dipole & 1272.4 & 0.1 & KICKER \\
\hline IP10 & 1277.95 & 0 & MARKER & Left Dipole & 1272.5 & 0.1 & KICKER \\
\hline OX10A9 & 1280.03 & 2.0828 & DRIFT & Left Dipole & 1272.6 & 0.1 & KICKER \\
\hline AP10P13G & 1280.03 & 0 & MARKER & Left Dipole & 1272.7 & 0.1 & KICKER \\
\hline OX10A8 & 1280.23 & 0.19812 & DRIFT & Left Dipole & 1272.8 & 0.1 & KICKER \\
\hline AP10P14G & 1280.23 & 0 & MARKER & Left Dipole & 1272.9 & 0.1 & KICKER \\
\hline OX10A7 & 1280.45 & 0.2159 & DRIFT & Left Dipole & 1273 & 0.1 & KICKER \\
\hline AP10P15G & 1280.45 & 0 & MARKER & Left Dipole & 1273.1 & 0.1 & KICKER \\
\hline OEDCERNFL & 1281.02 & 0.575056 & DRIFT & Left Dipole & 1273.2 & 0.1 & KICKER \\
\hline EDCERN & 1281.02 & 0 & DRIFT & Left Dipole & 1273.3 & 0.1 & KICKER \\
\hline OEDCERNFL & 1281.6 & 0.575056 & DRIFT & Left Dipole & 1273.4 & 0.1 & KICKER \\
\hline AP10P16G & 1281.6 & 0 & MARKER & Left Dipole & 1273.5 & 0.1 & KICKER \\
\hline OX10A6 & 1281.88 & 0.279908 & DRIFT & Left Dipole & 1273.6 & 0.1 & KICKER \\
\hline AP10P17G & 1281.88 & 0 & MARKER & YSOLEN & 1275.95 & 2.35 & SOLEN \\
\hline OEDCERNFL & 1282.45 & 0.575056 & DRIFT & Middle & 1276.05 & 0.1 & KICKER \\
\hline EDCERN & 1282.45 & 0 & DRIFT & Middle & 1276.15 & 0.1 & KICKER \\
\hline OEDCERNFL & 1283.03 & 0.575056 & DRIFT & Middle & 1276.25 & 0.1 & KICKER \\
\hline AP10P18G & 1283.03 & 0 & MARKER & Middle & 1276.35 & 0.1 & KICKER \\
\hline OX10A5 & 1283.24 & 0.2159 & DRIFT & Middle & 1276.45 & 0.1 & KICKER \\
\hline AP10P19G & 1283.24 & 0 & MARKER & Middle & 1276.55 & 0.1 & KICKER \\
\hline OX10A4 & 1283.44 & 0.19812 & DRIFT & Middle & 1276.65 & 0.1 & KICKER \\
\hline AP10P20G & 1283.44 & 0 & MARKER & Middle & 1276.75 & 0.1 & KICKER \\
\hline OX10A3 & 1285.36 & 1.92126 & DRIFT & Middle & 1276.85 & 0.1 & KICKER \\
\hline AP10P21G & 1285.36 & 0 & MARKER & Middle & 1276.95 & 0.1 & KICKER \\
\hline OX10A2 & 1285.69 & 0.333248 & DRIFT & Middle & 1277.05 & 0.1 & KICKER \\
\hline AP10P22G & 1285.69 & 0 & MARKER & Middle & 1277.15 & 0.1 & KICKER \\
\hline
\end{tabular}




\begin{tabular}{|c|c|c|c|c|c|c|c|}
\hline OX10A1 & 1286.08 & 0.390525 & DRIFT & Middle & 1277.25 & 0.1 & KICKER \\
\hline AP10P23G & 1286.08 & 0 & MARKER & Middle & 1277.35 & 0.1 & KICKER \\
\hline OBXFLX & 1286.27 & 0.1905 & DRIFT & Middle & 1277.45 & 0.1 & KICKER \\
\hline BPMH & 1286.27 & 0 & HBPM & Middle & 1277.55 & 0.1 & KICKER \\
\hline CPLMON & 1286.27 & 0 & BPM & Middle & 1277.65 & 0.1 & KICKER \\
\hline BPMV & 1286.27 & 0 & VBPM & Middle & 1277.75 & 0.1 & KICKER \\
\hline OBXFLA & 1286.47 & 0.1905 & DRIFT & Middle & 1277.85 & 0.1 & KICKER \\
\hline AP10P24G & 1286.47 & 0 & MARKER & Middle & 1277.95 & 0.1 & KICKER \\
\hline OX10A0 & 1286.51 & 0.04875 & DRIFT & IP10 & 1277.95 & 0 & MARKER \\
\hline MVALVE & 1286.51 & 0 & MARKER & Middle & 1278.05 & 0.1 & KICKER \\
\hline OBSK10LG & 1286.56 & 0.048623 & DRIFT & Middle & 1278.15 & 0.1 & KICKER \\
\hline AP10P25G & 1286.56 & 0 & MARKER & Middle & 1278.25 & 0.1 & KICKER \\
\hline ODXFLX & 1287.75 & 1.18563 & DRIFT & Middle & 1278.35 & 0.1 & KICKER \\
\hline ELDXMP10 & 1287.75 & 0 & DRIFT & Middle & 1278.45 & 0.1 & KICKER \\
\hline HDXMP10M0 & 1287.75 & 0 & MULT & Middle & 1278.55 & 0.1 & KICKER \\
\hline HDXMP10 & 1288.21 & 0.462527 & SBEND & Middle & 1278.65 & 0.1 & KICKER \\
\hline HDXMP10M1 & 1288.21 & 0 & MULT & Middle & 1278.75 & 0.1 & KICKER \\
\hline HDXMP10 & 1288.67 & 0.462527 & SBEND & Middle & 1278.85 & 0.1 & KICKER \\
\hline HDXMP10M2 & 1288.67 & 0 & MULT & Middle & 1278.95 & 0.1 & KICKER \\
\hline HDXMP10 & 1289.14 & 0.462527 & SBEND & Middle & 1279.05 & 0.1 & KICKER \\
\hline HDXMP10M3 & 1289.14 & 0 & MULT & Middle & 1279.15 & 0.1 & KICKER \\
\hline HDXMP10 & 1289.6 & 0.462527 & SBEND & Middle & 1279.25 & 0.1 & KICKER \\
\hline \multirow[t]{18}{*}{ HDXMP10M4 } & 1289.6 & 0 & MULT & Middle & 1279.35 & 0.1 & KICKER \\
\hline & & & & Middle & 1279.45 & 0.1 & KICKER \\
\hline & & & & Middle & 1279.55 & 0.1 & KICKER \\
\hline & & & & Middle & 1279.65 & 0.1 & KICKER \\
\hline & & & & Middle & 1279.75 & 0.1 & KICKER \\
\hline & & & & Middle & 1279.85 & 0.1 & KICKER \\
\hline & & & & Middle & 1279.95 & 0.1 & KICKER \\
\hline & & & & BSOLEN & 1282.3 & 2.35 & SOLEN \\
\hline & & & & Right Dipole & 1282.4 & 0.1 & KICKER \\
\hline & & & & Right Dipole & 1282.5 & 0.1 & KICKER \\
\hline & & & & Right Dipole & 1282.6 & 0.1 & KICKER \\
\hline & & & & Right Dipole & 1282.7 & 0.1 & KICKER \\
\hline & & & & Right Dipole & 1282.8 & 0.1 & KICKER \\
\hline & & & & Right Dipole & 1282.9 & 0.1 & KICKER \\
\hline & & & & Right Dipole & 1283 & 0.1 & KICKER \\
\hline & & & & Right Dipole & 1283.1 & 0.1 & KICKER \\
\hline & & & & Right Dipole & 1283.2 & 0.1 & KICKER \\
\hline & & & & Right Dipole & 1283.3 & 0.1 & KICKER \\
\hline
\end{tabular}




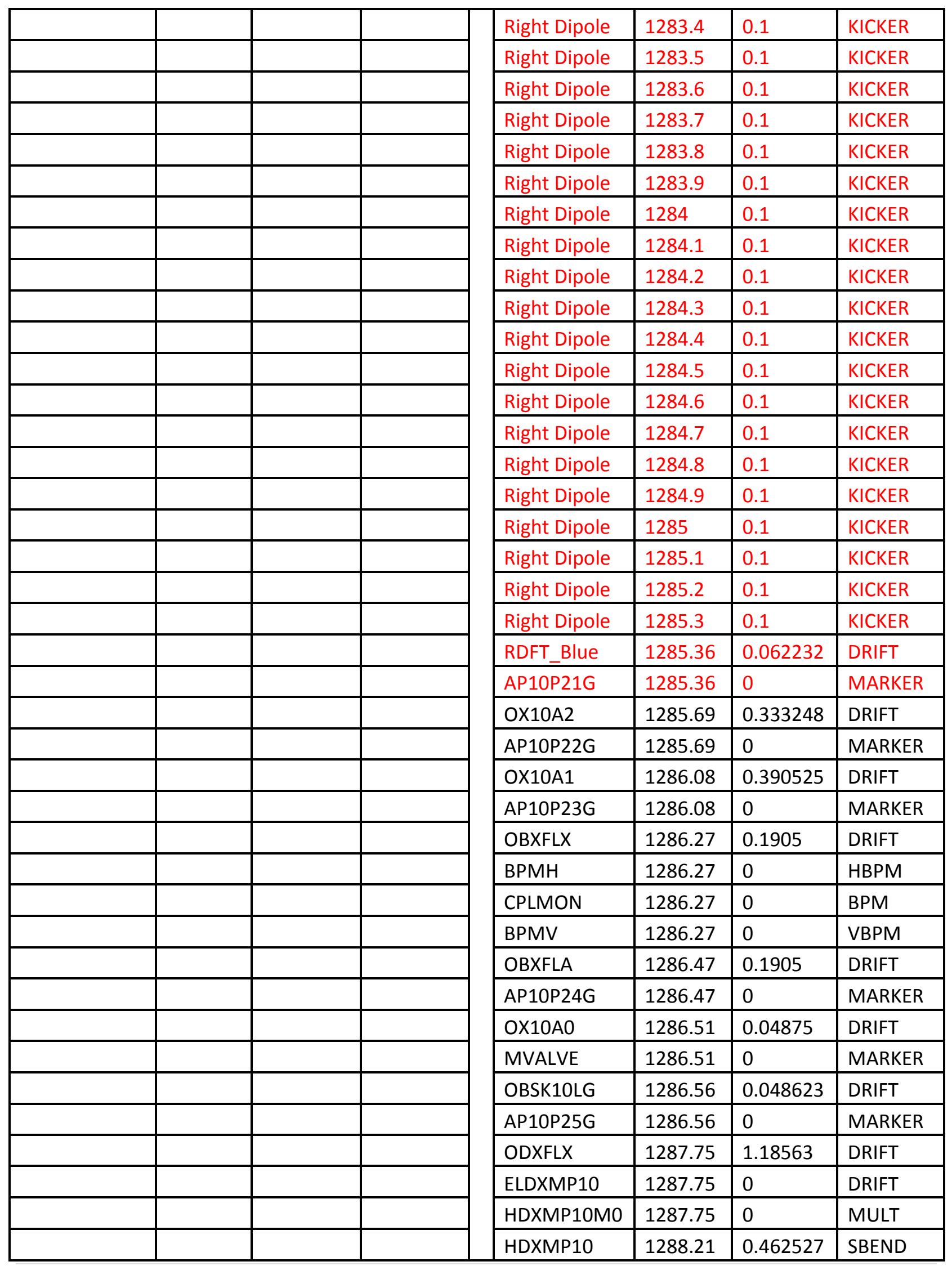




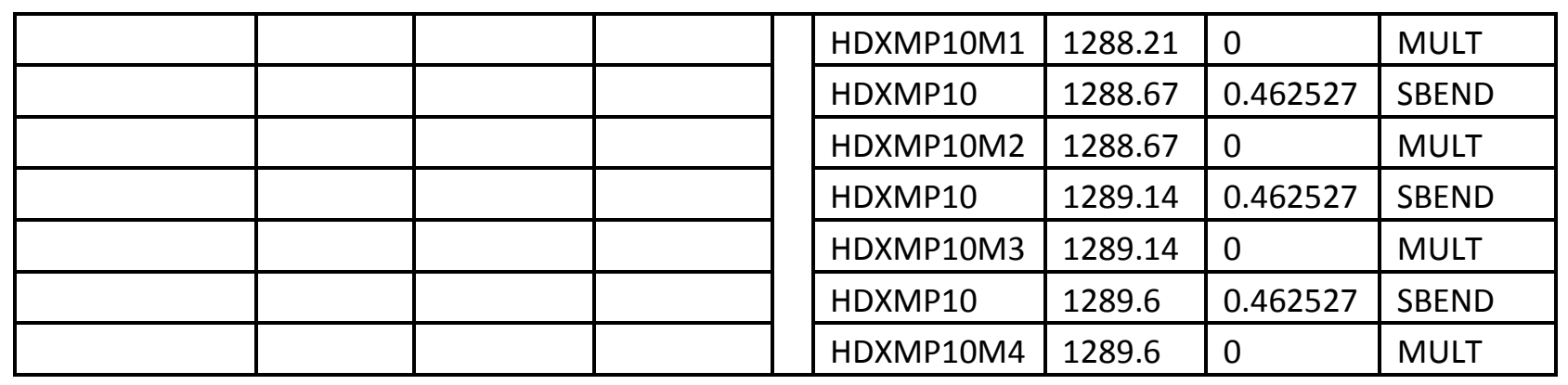




\section{Appendix B}
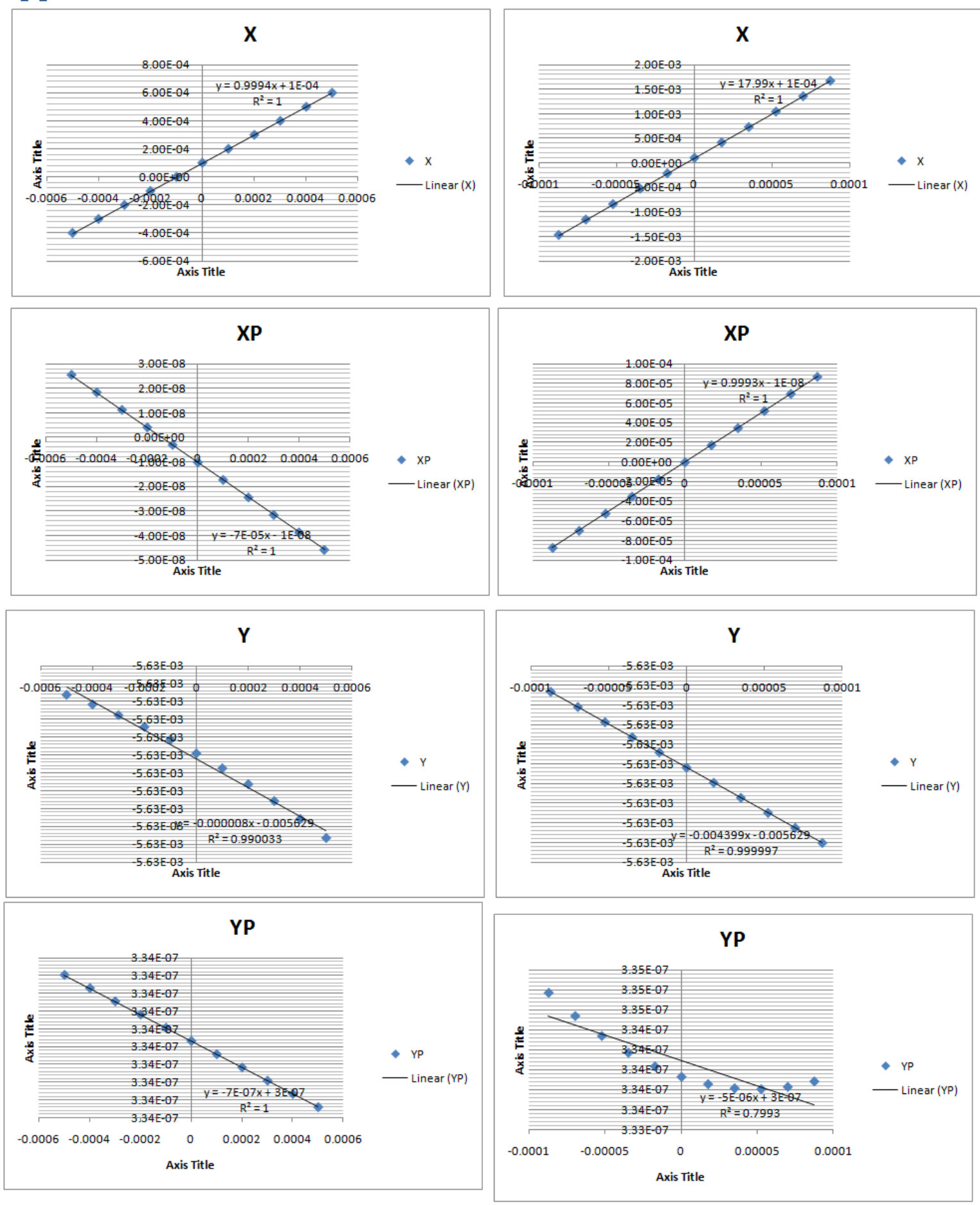

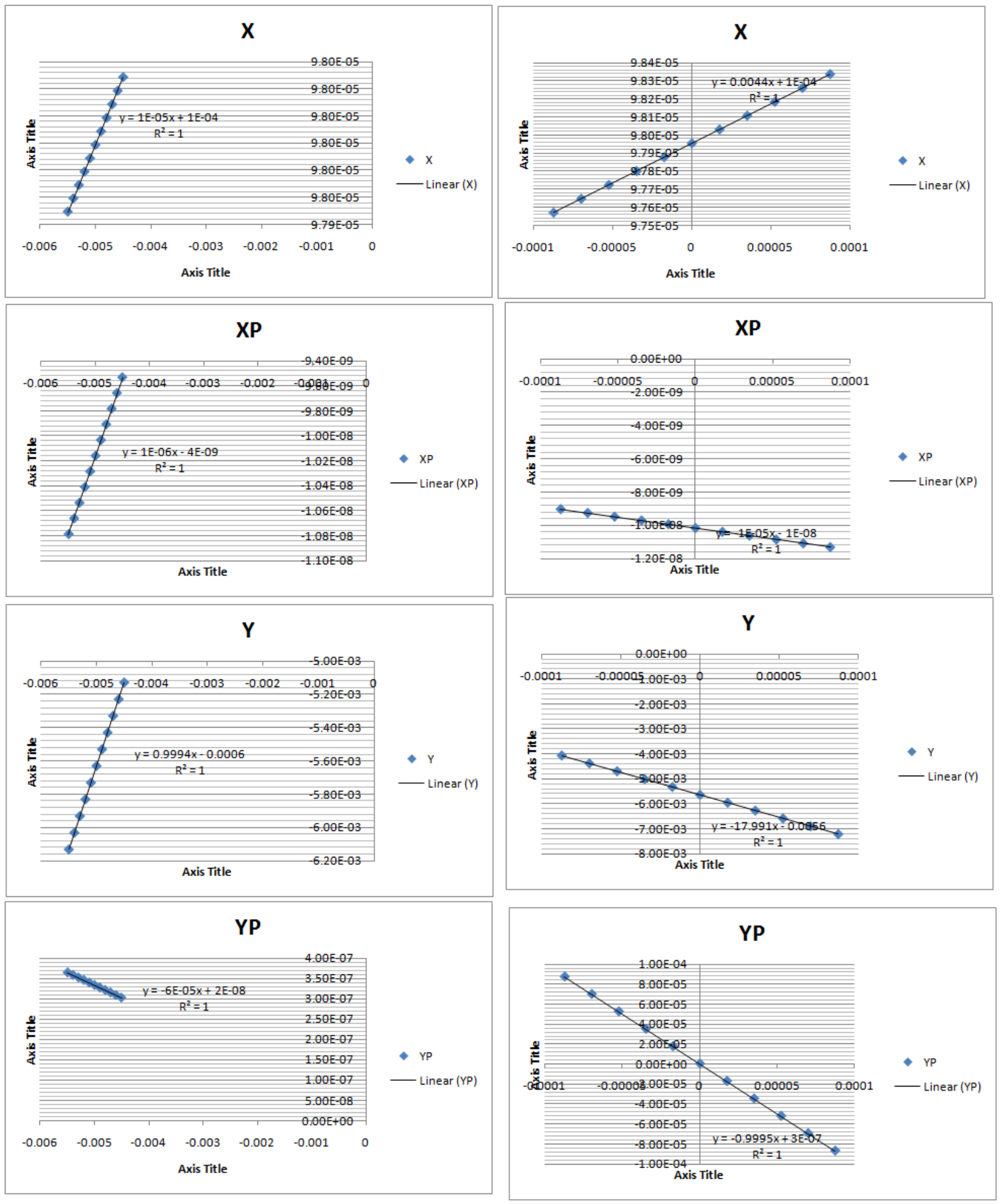

Figure 17: Fitting the function, and obtaining the coefficients. 